\title{
Bevacizumab for Progressive Vestibular Schwannoma in Neurofibromatosis Type 2
}

\section{Citation}

Plotkin, Scott R., Vanessa L. Merker, Chris Halpin, Dominique Jennings, Michael J. McKenna, Gordon J. Harris, and Fred G. Barker. 2012. "Bevacizumab for Progressive Vestibular Schwannoma in Neurofibromatosis Type 2." Otology \& Neurotology 33 (6) (August): 1046-1052. doi:10.1097/mao.0b013e31825e73f5.

\section{Published Version}

doi:10.1097/MA0.0b013e31825e73f5

\section{Permanent link}

http://nrs.harvard.edu/urn-3:HUL.InstRepos:33788498

\section{Terms of Use}

This article was downloaded from Harvard University's DASH repository, and is made available under the terms and conditions applicable to Other Posted Material, as set forth at http:// nrs.harvard.edu/urn-3:HUL.InstRepos:dash.current.terms-of-use\#LAA

\section{Share Your Story}

The Harvard community has made this article openly available.

Please share how this access benefits you. Submit a story.

\section{Accessibility}




\section{ABSTRACT}

Objective: Early studies suggest that bevacizumab treatment can result in tumor shrinkage and hearing improvement for some patients with neurofibromatosis 2 (NF2). The aim of this study was to report extended follow-up in a larger cohort of similarly treated patients.

Study Design: Retrospective study.

Setting: Tertiary referral center

Patients: 31 consecutive NF2 patients who received bevacizumab for progressive vestibular schwannomas.

Main Outcome Measure: Hearing improvement, defined as an improvement in word recognition score above the $95 \%$ critical difference compared with baseline, and radiographic response, defined as a $\geq 20 \%$ decrease in tumor volume compared with baseline.

Results: The median age was 26 years (range, 12-73). The median volumetric tumor growth rate before treatment was $64 \%$ per year. At the time of analysis, the median duration of treatment was 14 months (range, 6-41) with a total of 45 patient-years of follow up. A hearing response occurred in 13/23 (57\%) evaluable patients, and a radiographic response in 17/31 (55\%) of target vestibular schwannomas. The median time to response was 3 months for both endpoints. The only clinical or radiographic feature at baseline that correlated with change in tumor volume at 3 months was the mean apparent diffusion coefficient (ADC) value, a radiologic marker of edema $(\mathrm{p}=0.036) .90 \%$ of patients had stable or improved hearing after 1 year of treatment and $61 \%$ at 3 years. $88 \%$ of patients had stable or decreased tumor size after 1 year of treatment and $54 \%$ at 3 years. Overall, treatment was well tolerated.

Conclusion: Bevacizumab treatment was followed by hearing improvement and tumor shrinkage in over $50 \%$ of progressive vestibular schwannomas in NF2 patients. Stable or 
improved hearing was retained in the majority of patients. 


\section{INTRODUCTION}

Neurofibromatosis type 2 (NF2) is a hereditary tumor predisposition syndrome caused by mutations in the NF2 tumor suppressor gene. Individuals with NF2 have a higher likelihood to develop multiple nervous system tumors, including schwannomas, meningiomas, and ependymomas. ${ }^{1}$ The hallmark of NF2 is bilateral vestibular schwannomas. These tumors arise from the vestibular portion of cranial nerve VIII; over time, they lead to hearing loss, tinnitus, and imbalance. Historically, most NF2 patients experience complete hearing loss either from tumor progression or after treatment of the tumors with surgery or radiation. In unselected NF2 patients, the average linear growth rate of vestibular schwannomas is $1.9 \mathrm{~mm} /$ year. $^{2} \mathrm{NF} 2$ patients also experience progressive hearing loss over time. ${ }^{3}$ In NF2 patients with progressive vestibular schwannomas, the median times to hearing loss and volumetric tumor progression are 9 and 9.2 months, respectively, after treatment with erlotinib. ${ }^{4}$ Effective treatments are urgently needed for NF2 patients with progressive hearing loss, because hearing loss is associated with impairment in social, emotional, and communication function, and increased depression. ${ }^{5-7}$

Previous studies of NF2 patients treated with bevacizumab suggested that inhibition of vascular endothelial growth factor (VEGF) could result in hearing improvement and reduction in tumor size. ${ }^{8-10}$ However, the durability of these effects on hearing and tumor size is unclear. ${ }^{11}$ Therefore, we retrospectively addressed this question in a larger series of patients treated at a single institution with a total of 45 patient-years of follow up.

\section{MATERIALS AND METHODS}

\section{Study Cohort}


We offered bevacizumab on a compassionate-use basis to patients who fulfilled clinical diagnostic criteria for NF2, ${ }^{12 ; 13}$ had evidence of progressive vestibular schwannomas and were considered poor candidates for surgery and radiation therapy or refused these treatments. Consecutive patients who met these criteria and received at least one bevacizumab dose as of March 4, 2011, were included in the analysis. Seven patients were previously treated with erlotinib (patients 1-5 and 7-9). ${ }^{4}$ The annual tumor growth rate prior to treatment was calculated using the immediate pre-treatment MRI scan and scans obtained within 1 year before treatment.

\section{Treatment}

Baseline MRI and audiology were performed before starting treatment (median 1 month; range, $0.1-9$ months). Clinical evaluation including a physical exam, complete blood count, chemistry panel, and urinalysis was performed every 2 to 4 weeks during treatment. Tumor response was monitored using serial MRI scanning at clinical visits every 3 months. Tumor volumetric analysis was performed as described previously. ${ }^{14}$ To investigate whether tumor shrinkage might be related, in part, to a decrease in intratumoral vasogenic edema, we subsequently determined the mean apparent diffusion coefficient, a measure of the magnitude of diffusion of water molecules within tissue and a radiographic marker of edema within vestibular schwannomas at baseline. ${ }^{15}$

Hearing response was monitored using serial audiologic evaluations including determination of pure tone thresholds and word recognition scores. ${ }^{16 ; 17}$ Toxicity data were collected during routine clinic visits and were scored according to the National Cancer Institute's Common Terminology Criteria for Adverse Events, version 4.0. The institutional review board at Massachusetts General Hospital approved the retrospective chart review. All patients provided written informed consent for treatment. 


\section{Criteria for hearing improvement and radiographic responses}

Hearing improvement was defined as a statistically significant increase in word recognition score $(\mathrm{p}<0.05)$ compared to the baseline score. ${ }^{18}$ Patients were eligible for hearing improvement if their pretreatment word recognition score was $\leq 94 \%$ on a 50 -word list (i.e., if their baseline hearing allowed for a measurable increase above the upper limit of the critical difference threshold). Patients with surgically absent auditory nerves were not eligible for hearing improvement. Differences between word recognition scores at baseline and during treatment were compared using published tables for word recognition scores ${ }^{19}$ and the American Academy of Otolaryngology-Head and Neck Surgery (AAO-HNS) Committee on Hearing and Equilibrium classification. ${ }^{20}$ Radiographic response was defined as a decrease in tumor volume of $20 \%$ or more compared to the pretreatment baseline. ${ }^{21}$

\section{Criteria for progressive hearing loss and radiographic progression}

Hearing loss was defined as a statistically significant decrease in word recognition score $(\mathrm{p}<0.05)$ compared to the baseline either due to tumor progression or to surgery. ${ }^{18}$ Patients were eligible for progressive hearing loss if their pretreatment word recognition score was $\geq 6 \%$ on a 50-word list (i.e., allowed for a measurable decrease below the lower limit of the critical difference threshold). Radiographic progression was defined as either (1) an increase in tumor volume of $20 \%$ or more on the most recent MRI study compared to the pretreatment baseline, or (2) surgery for the target vestibular schwannoma. ${ }^{21}$ Survival times were calculated from the start of bevacizumab treatment until analysis was closed on 4/1/2011. Tumor size and hearing function in patients on drug holidays due to adverse events, surgical treatment of non-vestibular tumors, or insurance coverage issues were included in the analysis. 


\section{Statistical Analyses}

We calculated descriptive statistics for clinical and demographic factors for the study group. We used ordinal logistic regression to test for factors predicting hearing response or radiographic response (best response, coded as response/stable/progression). Continuous variables were coded as greater than vs. less than or equal to the median during regression analysese. We used Kaplan Meier methods to estimate time to hearing loss and time to radiographic progression and Cox proportional hazards regression to test for factors predicting loss of hearing or radiographic progression. All statistical calculations were performed using the statistical programming language R Version 2.12 (R Development Core Team, Vienna, Austria). ${ }^{22}$

\section{RESULTS}

\section{Study Cohort and Treatment}

The baseline clinical characteristics of the 31 consecutive patients we treated using bevacizumab for NF2-related vestibular schwannoma are shown in Table 1 and Supplemental Digital Content 1. Twenty-five patients were at risk for hearing loss in their only (or better) hearing ear as the indication for starting treatment, and 6 were at risk for critical brainstem compression. All patients were poor candidates for standard treatment or refused these treatments. Fourteen men and seventeen women with a median age of 26 years (range, 12 to 73 years) received bevacizumab $5 \mathrm{mg} / \mathrm{kg}$ IV every 2 weeks. The median annual tumor volume growth rate before treatment was $64 \%$ (range, $-19 \%$ to $240 \%$ ). The median duration of treatment at the time of analysis was 14 months (range, 6 to 41 months) with twenty patients followed for at least one year (Fig. 1) and 25 patients still receiving treatment at the time of analysis. Six 
patients stopped therapy for clinical progression of the target VS or other NF2-related tumor after a median of 11.5 months (range, 3 - 18 months).

\section{Radiologic and Hearing Responses}

A total of 31 target vestibular schwannomas and 20 contralateral vestibular schwannomas could be evaluated for radiologic response. Most vestibular schwannomas had a detectable decrease in tumor size (Fig. 2A). The overall radiologic response rate (i.e., a decrease in tumor volume of $20 \%$ or more compared to baseline) was $53 \%$ (27/51, Figure $2 \mathrm{~A})$. For the target vestibular schwannomas, the radiologic response rate was 55\% (17/31) and for contralateral vestibular schwannomas the rate was 50\% (10/20). Of 27 radiologic responses, 18 were confirmed on subsequent imaging. Of the 9 tumors without a confirmed response, 5 showed a transient decrease in volume of $20 \%$ with subsequent growth, 2 had a response at their most recent imaging and have not yet had confirmatory imaging, and 2 tumors had only 1 posttreatment scan before undergoing surgery for an unrelated tumor. The median time to radiologic response was 3 months, with $94 \%$ of responding target tumors and $60 \%$ of responding non-target tumors having a response by 3 months (Figure 2B). Overall, 17/19 (89\%) of responding patients had a response by 3 months in at least one tumor. There was a significant correlation between radiographic response in the target vestibular schwannoma and the contralateral schwannoma ( $\mathrm{P}$ $<0.05)$.

Among the six patients at risk for critical brainstem compression, the radiographic response rate of the target VS was 50\%. During treatment, 4 patients experienced progressive tumor growth of a target VS (3 patients) or other NF2-related tumor (1 patient). Bevacizumab was discontinued in 3 patients and was followed by surgery after 1.7, 2.9, and 3.2 months. There 
were no post-operative complications related to bevacizumab use but two patients died after surgery $(2.0,2.9$ months $)$ due to post-operative medical complications.

A total of 23 target vestibular schwannomas and 5 contralateral vestibular schwannomas could be evaluated for hearing response. Two patients were not eligible for hearing response because they did not have auditory nerves due to previous surgery, and six patients were ineligible for hearing improvement due to baseline word recognition score of $94 \%$ or greater. Most patients had some increase in word recognition score (Fig. 2C). The overall hearing response rate was $57 \%(16 / 28)$. For the target ears, the hearing response rate was $58 \%(13 / 23)$ and for contralateral ears the rate was $60 \%(3 / 5)$. Of three people with word recognition scores of $0 \%$ prior to treatment, two developed measurable scores: one patient's score peaked at $42 \%$ and the other peaked at $28 \%$. Of 16 hearing responses, 14 were confirmed on subsequent audiology. Of the 2 patients without a confirmed hearing response, one achieved a response, but was measured on only one audiogram before treatment was held for insurance issues. During the drug holiday, word recognition declined; after treatment resumed, the patient again achieved a hearing response on the first audiogram after resuming treatment. The other patient had multiple audiograms during treatment but did not have a second study that confirmed the hearing response. The median time to hearing response was 3 months, with $85 \%$ of responding target ears and 33\% of responding non-target ears having a response by 3 months. Overall, 12/16 (75\%) responding patients had a response by 3 months in at least one ear.

Twenty-nine tumors in 23 patients were evaluable for both hearing and radiographic response. There was no correlation between hearing response and radiographic response, either when analyzed per patient or per tumor $(\chi 2=0.11, \mathrm{p}=0.74)$. 


\section{Predictors of response}

We tested the following clinical factors as predictors of best radiologic and hearing response: age, gender, baseline tumor volume, baseline annual growth rate, baseline AAO-HNS hearing classification, and baseline word recognition score. None of these clinical factors correlated significantly with either radiologic or hearing response $(\mathrm{P}>0.05)$. Five patients received radiation and 16 patients received other chemotherapy prior to treatment with bevacizumab. The median time between radiation therapy and the start of bevacizumab treatment was 11 years (range, 2 to 13 years). The radiologic response rate was 71\% (5/7) for radiated tumors and was $59 \%(16 / 27)$ for tumors treated with previous chemotherapy. Four evaluable tumors in four patients received radiation therapy to a target vestibular schwannoma prior to bevacizumab. In this group, a hearing response was noted in 3 patients $(75 \%)$.

Baseline apparent diffusion coefficient (ADC) values for tumors were available for 29 target schwannomas and 16 contralateral schwannomas. The mean ADC value of a tumor at baseline correlated modestly with subsequent change in tumor volume at 3 months (Figure 3 ). This effect was strongest for target vestibular schwannomas $(\mathrm{N}=29, \mathrm{r}=-0.458, \mathrm{p}=0.016)$, but was also present when considering all vestibular schwannomas $(\mathrm{N}=45, \mathrm{r}=-0.32, \mathrm{p}=0.036)$.

\section{Time to tumor growth}

All 31 patients were evaluable for radiologic progression in the target ear. Seven target tumors progressed during treatment, including 3 with tumor growth and 4 who required surgery for the target vestibular schwannoma. The rates of tumor stability or shrinkage (i.e., freedom from tumor growth or surgery) at last follow up were $88 \%$ at 1 year, $67 \%$ at 2 years, and $54 \%$ at 3 years (Fig. 4A). The median time to tumor growth was not reached by the time of analysis. Three patients with stable disease at last follow up experienced fluctuations in tumor volume 
during treatment, with occasional values more than $20 \%$ larger than baseline. These 3 patients were maintained on bevacizumab by physician discretion due to favorable hearing response during treatment.

\section{Time to hearing loss}

A total of 26 patients were evaluable for hearing loss in the target ear; the remaining five patients had no measurable hearing at baseline. Four patients experienced hearing loss in the target ear during treatment, including 3 patients with hearing loss on bevacizumab and one who lost hearing from surgery for a progressive tumor. Overall, the rates of hearing stability or improvement (i.e., freedom from hearing loss for any reason) at last follow up were $90 \%$ at 1

year, $81 \%$ at 2 years, and $61 \%$ at 3 years (Fig. $4 \mathrm{~b}$ ). The median time of hearing preservation was not reached by the time of analysis. The AAO-HNS hearing classification for patients before treatment and at last follow up is shown in Supplemental Digital Content 2. Six of $31(19 \%)$ patients moved up at least one class during treatment, 20/31 (65\%) did not change class, and 5/31 $(16 \%)$ declined one class.

\section{Drug interruptions}

There were 17 drug interruptions lasting longer than one month in 12 patients. The reasons for drug interruptions included surgery for a non-vestibular tumor (6), adverse event (6, including 1 hypertension, 2 proteinuria, 2 menorrhagia, and 1 pneumonia), loss of insurance coverage (3), and patient preference (2). The median length of drug interruption was 3.2 months (range, $1.6-15.1$ months). The median growth in tumor volume for a target tumor during drug interruption was $9.3 \%$ overall (range, $-2.5 \%$ to $46.1 \%$ ), or $2.6 \%$ per month (range, $-1.4 \%$ to 12.1\%). Six interruptions occurred in patients with a contralateral vestibular schwannoma; the median growth for contralateral tumor was $12.0 \%$ overall and $2.5 \%$ per month. Fifteen drug 
interruptions occurred in patients evaluable for hearing loss. Hearing significantly declined during 4 interruptions in 4 separate patients, and remained stable during 9 interruptions in 8 patients. Hearing could not be measured during two interruptions due to the timing of audiology assessments.

\section{Adverse Events}

We identified 168 adverse events during 572 patient-months of treatment (Table 2). There were 134 grade 1 adverse events (79\%), 26 grade 2 events (15\%), 8 grade 3 events (5\%) and 1 grade 4 event (1\%). The most common adverse events were menorrhagia (50\%) and irregular menses (50\%) in pre-menopausal women and hypertension (35\%) and proteinuria (35\%) in all patients. Grade 2 proteinuria led to dose interruption in 3 of 31 patients (11\%); all subsequently restarted treatment after proteinuria improved. Seven patients (23\%) noted symptoms associated with bevacizumab infusion including fatigue (5), nausea (1), headache (1), and abdominal discomfort (1). There were no intracranial hemorrhages or treatment-related deaths.

\section{DISCUSSION}

The results of this study agree with previous estimates of the activity of bevacizumab against progressive vestibular schwannoma in NF2 patients. ${ }^{8 ; 10 ; 23 ; 24}$ In this larger cohort of 31 patients with 52 vestibular schwannomas and a total of 45 patient-years of follow up, the rates of hearing improvement (54\%) and tumor shrinkage (57\%) during bevacizumab treatment were

similar to an earlier report. ${ }^{8}$ The radiographic and hearing response rate were similar for target vestibular schwannomas (i.e., the progressive tumor at baseline) and for contralateral vestibular 
schwannomas. Clinical responses to treatment were rapid with most patients achieving a response after 3 months.

Ideally, clinicians would like to be able to select patients who are likely to respond to bevacizumab treatment. The only clinical or radiographic feature that was associated with a radiographic response was the mean $\mathrm{ADC}$ value of the tumor at baseline. This association, though weaker than reported previously ${ }^{8}$, supports the notion that leaky tumor blood vessels result in increased water content within the tumor. No other clinical factors were associated with a hearing response. In addition, there was no significant correlation between hearing response and radiographic response in target tumors, raising the possibility that different biological processes are responsible for hearing improvement and tumor shrinkage. Discordance between hearing loss and tumor size in vestibular schwannoma has been reported previously.. ${ }^{25}$

The durability of clinical response to bevacizumab in NF2 patients is notable and contrasts with previous reports in malignant tumors. Modest increases in progression-free survival have been reported with the addition of bevacizumab for treatment of many solid tumor types including breast cancer, colon cancer, and glioblastoma, but disease progression remains the rule ${ }^{26-30}$ The median times to hearing loss and tumor progression were not reached in this study and exceed 3 years (Fig. 3), at which time freedom from hearing loss and tumor growth were $61 \%$ and $54 \%$, respectively. Although the durability of response in NF2 patients is not explained in the current study, it may reflect the benign histology of vestibular schwannomas and the absence of genetic instability that is characteristic of malignant tumors.

Unplanned drug interruptions were relatively common in this study. Discontinuation of bevacizumab was associated with an increase in tumor volume and a decline in hearing in some patients. This finding agrees with previous studies in animal models in which discontinuation of 
anti-VEGF therapy results in regrowth of tumor vasculature and acceleration of metastasis..$^{31 ; 32}$ It does not agree with retrospective analysis of randomized placebo-controlled phase 3 studies in solid tumors in which discontinuation of bevacizumab did not result in decreased time to disease progression. ${ }^{33}$ At this time, the optimal duration of bevacizumab treatment for NF2 patients is not known. Overall, treatment was well tolerated: no patients discontinued treatment due to side effects. In addition, the safety profile in this cohort was favorable with low rates of grade 3 or 4 toxicity. Given the recent report of premature ovarian insufficiency in pre-menopausal women treated with chemotherapy plus bevacizumab ${ }^{34}$, close attention to the potential for long term toxicity is warranted in this patient population that tends to be younger than patients with cancer. 


\section{Reference List}

1. Evans DG, Sainio M, Baser ME. Neurofibromatosis type 2. J Med Genet 2000;37:897-904.

2. Slattery WH, III, Fisher LM, Iqbal Z et al. Vestibular schwannoma growth rates in neurofibromatosis type 2 natural history consortium subjects. Otol Neurotol 2004;25:811817.

3. Masuda A, Fisher LM, Oppenheimer ML et al. Hearing changes after diagnosis in neurofibromatosis type 2. Otol Neurotol 2004;25:150-154.

4. Plotkin SR, Halpin C, McKenna MJ et al. Erlotinib for progressive vestibular schwannoma in neurofibromatosis 2 patients. Otol Neurotol 2010;31:1135-1143.

5. Mulrow CD, Aguilar C, Endicott JE et al. Quality-of-life changes and hearing impairment. A randomized trial. Ann Intern Med 1990;113:188-194.

6. Kramer S. Hearing impairment, work, and vocational enablement. Int J Audiol Suppl 2008;2:S124-130.

7. Chia EM, Wang JJ, Rochtchina E et al. Hearin impairment and health-related quality of life: the Blue Mountains Hearing Study. Ear Hear 2007;28:187-195.

8. Plotkin SR, Stemmer-Rachamimov AO, Barker FG et al. Hearing improvement after bevacizumab in patients with neurofibromatosis type 2. N Engl J Med 2009;361:358-367.

9. Mautner VF, Nguyen $\mathrm{R}$, Kutta $\mathrm{H}$ et al. Bevacizumab induces regression of vestibular schwannomas in patients with neurofibromatosis type 2. Neuro Oncol 2010;12:14-18.

10. Eminowicz GK, Raman R, Conibear J et al. Bevacizumab treatment for vestibular schwannomas in neurofibromatosis type two: report of two cases, including responses after prior gamma knife and vascular endothelial growth factor inhibition therapy. $J$ Laryngol Otol 2011;126:79-82.

11. Mautner VF, Nguyen R, Knecht R et al. Radiographic regression of vestibular schwannomas induced by bevacizumab treatment: sustain under continuous drug application and rebound after drug discontinuation. Ann Oncol 2010;21:2294-2295.

12. Mulvihill JJ, Parry DM, Sherman JL et al. NIH conference. Neurofibromatosis 1 (Recklinghausen disease) and neurofibromatosis 2 (bilateral acoustic neurofibromatosis). An update. Ann Intern Med 1990;113:39-52.

13. Baser ME, Friedman JM, Wallace AJ et al. Evaluation of clinical diagnostic criteria for neurofibromatosis 2. Neurology 2002;59:1759-1765.

14. Harris GJ, Plotkin SR, MacCollin M et al. Three-dimensional volumetrics for tracking vestibular schwannoma growth in neurofibromatosis type II. Neurosurgery 2008;62:13141319. 
15. Batchelor TT, Sorensen AG, di Tomaso E et al. AZD2171, a pan-VEGF receptor tyrosine kinase inhibitor, normalizes tumor vasculature and alleviates edema in glioblastoma patients. Cancer Cell 2007;11:83-95.

16. Monsell EM. New and revised reporting guidelines from the Committee on Hearing and Equilibrium. American Academy of Otolaryngology-Head and Neck Surgery Foundation, Inc. Otolaryngol Head Neck Surg 1995;113:176-178.

17. Hirsch IJ, DAVIS H, SILVERMAN SR et al. Development of materials for speech audiometry. J Speech Hear Disord 1952;17:321-337.

18. Thornton AR, Raffin MJ. Speech-discrimination scores modeled as a binomial variable. $J$ Speech Hear Res 1978;21:507-518.

19. Halpin C, Rauch SD. Using audiometric thresholds and word recognition in a treatment study. Otol Neurotol 2006;27:110-116.

20. Committee on Hearing and Equilibrium guidelines for the evaluation of hearing preservation in acoustic neuroma (vestibular schwannoma). American Academy of Otolaryngology-Head and Neck Surgery Foundation, INC. Otolaryngol Head Neck Surg 1995;113:179-180.

21. Plotkin SR, Halpin C, Blakely JO et al. Suggested response criteria for phase II antitumor drug studies for neurofibromatosis type 2 related vestibular schwannoma. J Neurooncol 2009;93:61-77.

22. R Development Core Team. R: a language and environment for statistical computing. 2008. Vienna, R Foundation for Statistical Computing.

23. Mautner VF, Nguyen R, Kutta H et al. Bevacizumab induces regression of vestibular schwannomas in patients with neurofibromatosis type 2. Neuro Oncol 2010;12:14-18.

24. Mautner VF, Nguyen R, Knecht R et al. Radiographic regression of vestibular schwannomas induced by bevacizumab treatment: sustain under continuous drug application and rebound after drug discontinuation. Ann Oncol 2010;21:2294-2295.

25. Fisher LM, Doherty JK, Lev MH et al. Concordance of bilateral vestibular schwannoma growth and hearing changes in neurofibromatosis 2: neurofibromatosis 2 natural history consortium. Otol Neurotol 2009;30:835-841.

26. Gray R, Bhattacharya S, Bowden C et al. Independent review of E2100: a phase III trial of bevacizumab plus paclitaxel versus paclitaxel in women with metastatic breast cancer. $J$ Clin Oncol 2009;27:4966-4972.

27. Brufsky AM, Hurvitz S, Perez E et al. RIBBON-2: A Randomized, Double-Blind, PlaceboControlled, Phase III Trial Evaluating the Efficacy and Safety of Bevacizumab in 
Combination With Chemotherapy for Second-Line Treatment of Human Epidermal Growth Factor Receptor 2-Negative Metastatic Breast Cancer. J Clin Oncol 2011;29:4286-4293.

28. Allegra CJ, Yothers $\mathrm{G}, \mathrm{O}^{\prime} \mathrm{C}$ onnell $\mathrm{MJ}$ et al. Phase III trial assessing bevacizumab in stages II and III carcinoma of the colon: results of NSABP protocol C-08. J Clin Oncol 2011;29:11-16.

29. Kreisl TN, Kim L, Moore K et al. Phase II trial of single-agent bevacizumab followed by bevacizumab plus irinotecan at tumor progression in recurrent glioblastoma. J Clin Oncol 2009;27:740-745.

30. Friedman HS, Prados MD, Wen PY et al. Bevacizumab alone and in combination with irinotecan in recurrent glioblastoma. J Clin Oncol 2009;27:4733-4740.

31. Mancuso MR, Davis R, Norberg SM et al. Rapid vascular regrowth in tumors after reversal of VEGF inhibition. J Clin Invest 2006;116:2610-2621.

32. Ebos JM, Lee CR, Cruz-Munoz W et al. Accelerated metastasis after short-term treatment with a potent inhibitor of tumor angiogenesis. Cancer Cell 2009;15:232-239.

33. Miles D, Harbeck N, Escudier B et al. Disease course patterns after discontinuation of bevacizumab: pooled analysis of randomized phase III trials. J Clin Oncol 2011;29:83-88.

34. U.S.Food and Drug Administration. Changes in Bevacizumab Labeling. Available at: http://www.accessdata.fda.gov/drugsatfda docs/label/2011/125085s225lbl.pdf. Accessed October 4,2011. 
Table 1. Baseline demographics and clinical characteristics of patients $(\mathrm{N}=31)$.

\begin{tabular}{|l|c|}
\hline Characteristic & \\
\hline Male sex-no. (\%) & $14(45 \%)$ \\
\hline Median age (range)--years & $26(12-73)$ \\
\hline Inheritance (sporadic)—no.(\%) & $27(87 \%)$ \\
\hline Previous radiotherapy to vestibular schwannoma—no. (\%) & $5(16 \%)$ \\
\hline Previous chemotherapy—no. (\%) & $16(52 \%)$ \\
\hline Indication for treatment—no. (\%) & $25(81 \%)$ \\
\hline Hearing Loss & $6(19 \%)$ \\
\hline Brainstem Compression & $9.6(1.3-38.7)$ \\
\hline Median baseline tumor size (range)—ml & \\
\hline Median baseline annual growth rate (range)—\% per year & $64 \%(-19 \%-240 \%)$ \\
\hline Target vestibular schwannoma & $32 \%(-85 \%-576 \%)$ \\
\hline Contralateral vestibular schwannoma & $4(13 \%)$ \\
\hline Hearing Status at baseline-no. (\%) & $22(71 \%)$ \\
\hline Bilateral Hearing Present & $5(16 \%)$ \\
\hline Unilateral Hearing Present & \\
\hline No Hearing Present & $8(26 \%)$ \\
\hline AAO-HNS hearing classification-no. (\%)* & $6(19 \%)$ \\
\hline Class A & $3(10 \%)$ \\
\hline Class B & $14(45 \%)$ \\
\hline Class C & \\
\hline Class D & \\
\hline
\end{tabular}

*AAO-HNS, American Academy of Otolaryngology-Head and Neck Surgery Committee on Hearing and Equilibrium(20) 
Table 2. Most common adverse events and laboratory abnormalities

\begin{tabular}{|l|c|c|c|c|}
\hline \multicolumn{1}{|c|}{ Adverse Event } & \multicolumn{2}{c|}{ Overall } & \multicolumn{2}{c|}{ Grade 3/4 } \\
\hline Hypertension & No. & $\%$ & No. & \% \\
\hline Proteinuria & 11 & $35 \%$ & 0 & 0 \\
\hline Epistaxis & 11 & $35 \%$ & 0 & $0 \%$ \\
\hline Menorraghia* & 8 & $26 \%$ & 2 & $6 \%$ \\
\hline Irregular menstruation* & 7 & $50 \%$ & 2 & $6 \%$ \\
\hline Fatigue & 7 & $50 \%$ & 0 & $0 \%$ \\
\hline Hyponatremia & 7 & $23 \%$ & 0 & $0 \%$ \\
\hline Leukopenia & 3 & $23 \%$ & 0 & $0 \%$ \\
\hline Rhinitis/nasal congestion & 3 & $10 \%$ & 0 & $0 \%$ \\
\hline $\begin{array}{l}\text { Wound Healing } \\
\text { Complication }\end{array}$ & 2 & $6 \%$ & & $0 \%$ \\
\hline Alopecia & 2 & $6 \%$ & 0 & $0 \%$ \\
\hline $\begin{array}{l}\text { Vascular access } \\
\text { complication }\end{array}$ & 1 & $3 \%$ & 1 & $3 \%$ \\
\hline $\begin{array}{l}\text { Gingival bleeding (oral } \\
\text { hemorrhage) }\end{array}$ & 1 & $3 \%$ & 0 & $0 \%$ \\
\hline Nasal Septum Perforation & 1 & $3 \%$ & & $0 \%$ \\
\hline Anorexia & 1 & $3 \%$ & 0 & $0 \%$ \\
\hline Headache & 1 & $3 \%$ & 0 & $0 \%$ \\
\hline Nausea/gastrointestinal pain & 1 & $3 \%$ & 0 & $0 \%$ \\
\hline Neutropenia & 1 & $3 \%$ & 1 & $3 \%$ \\
\hline Papilledema & 4 & $13 \%$ & 0 & $0 \%$ \\
\hline Reynaud's phenomenon & 3 & $10 \%$ & 0 & $0 \%$ \\
\hline Keratitis & 1 & $3 \%$ & 0 & $0 \%$ \\
\hline Skin infection & & $3 \%$ & 0 & 0 \\
\hline
\end{tabular}

(*includes 14 women who were menstruating at baseline). 
Figure 1. Duration of bevacizumab therapy and best radiologic response of the target vestibular schwannoma for the 31 patients in the study.

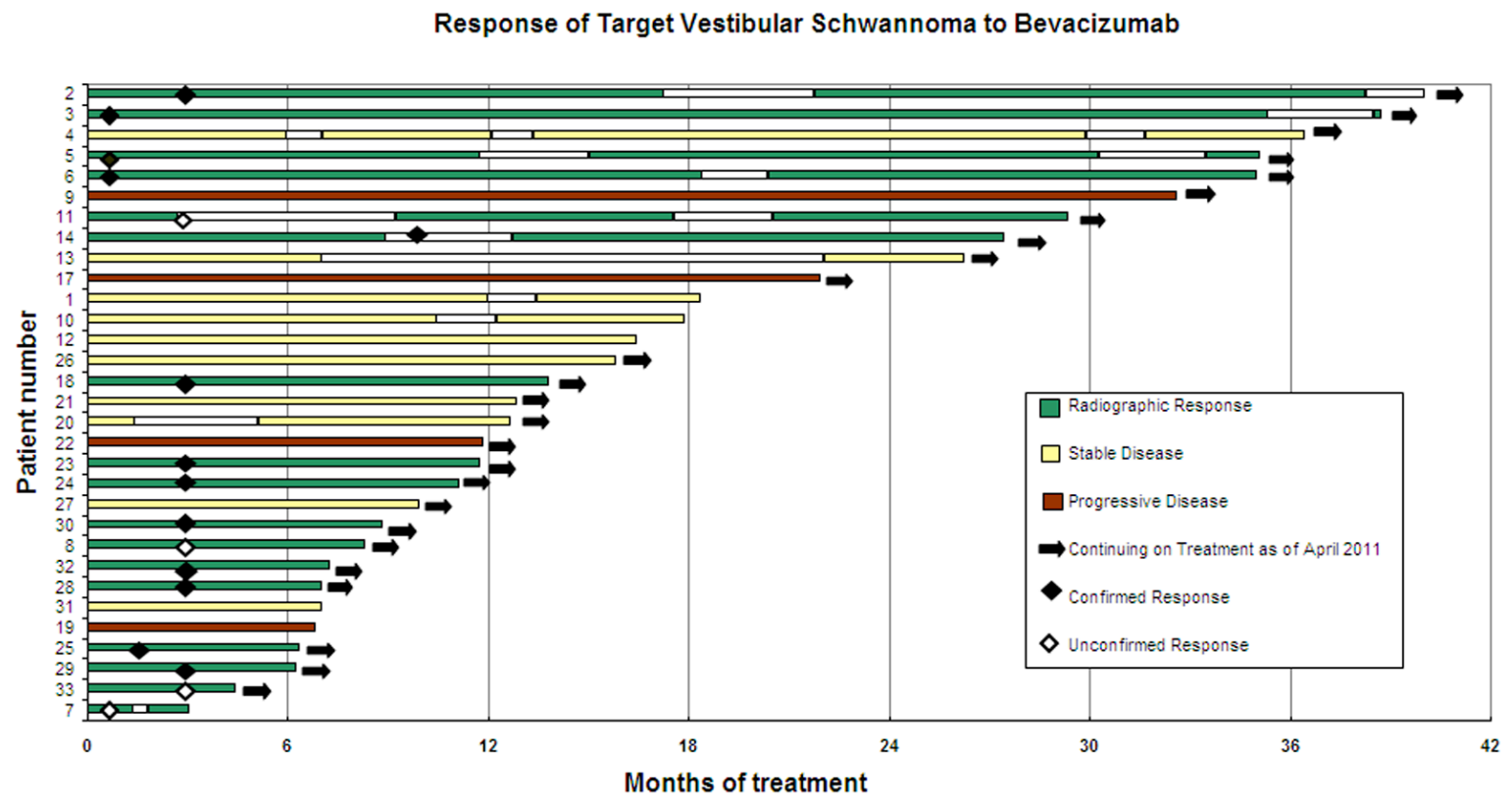

Each horizontal bar represents an individual patient. Green bars represent patients with radiologic responses, yellow bars represent patients with stable disease, and red bars represent patients with radiologic progression. White areas for each patient correspond to a drug interruption. Open diamonds represent unconfirmed radiologic responses, filled diamonds represent confirmed radiologic responses. 
Figure 2a. Best tumor response for each vestibular schwannoma.

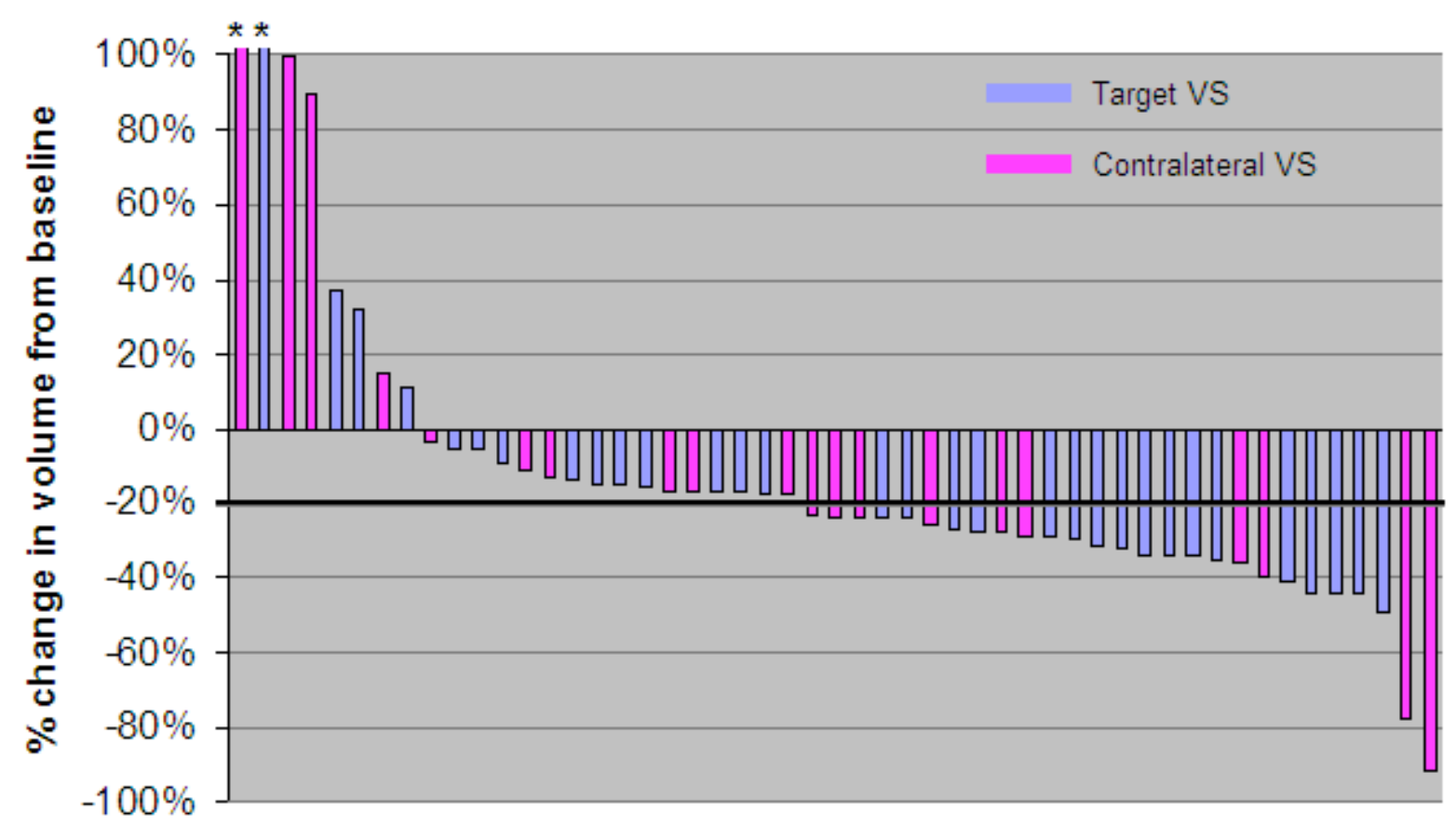

The best tumor responses (compared to baseline) are shown for the 31 target vestibular schwannomas (blue bars) and for the 20 contralateral vestibular schwannomas (green bars).

Each bar represents an individual vestibular schwannoma. The percent change in tumor volume from baseline is shown on the $\mathrm{Y}$-axis. Negative values indicate tumor shrinkage. * indicate $>$ $100 \%$ growth in volume 
Figure 2b. Change in tumor volume over time from a normalized baseline of $100 \%$.

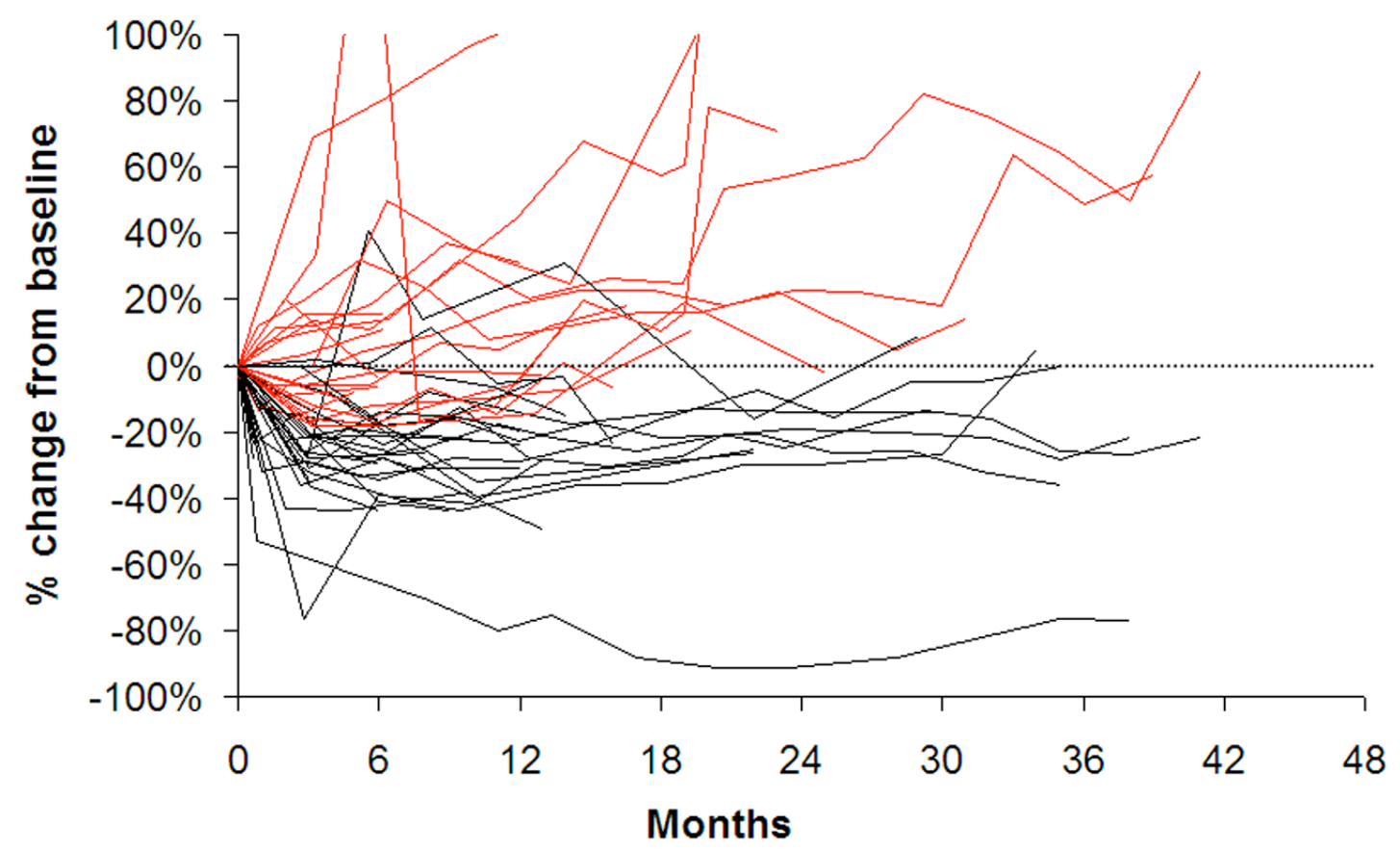

The change in tumor volume over time compared with baseline are shown for the 31 target vestibular schwannomas and 20 contralateral vestibular schwannomas. Tumors with radiographic responses are represented by black lines and tumors without radiographic responses are represented by red lines. The majority of responses occurred by 6 months and were durable in nature. 
Figure 2c. Best hearing response for each ear.

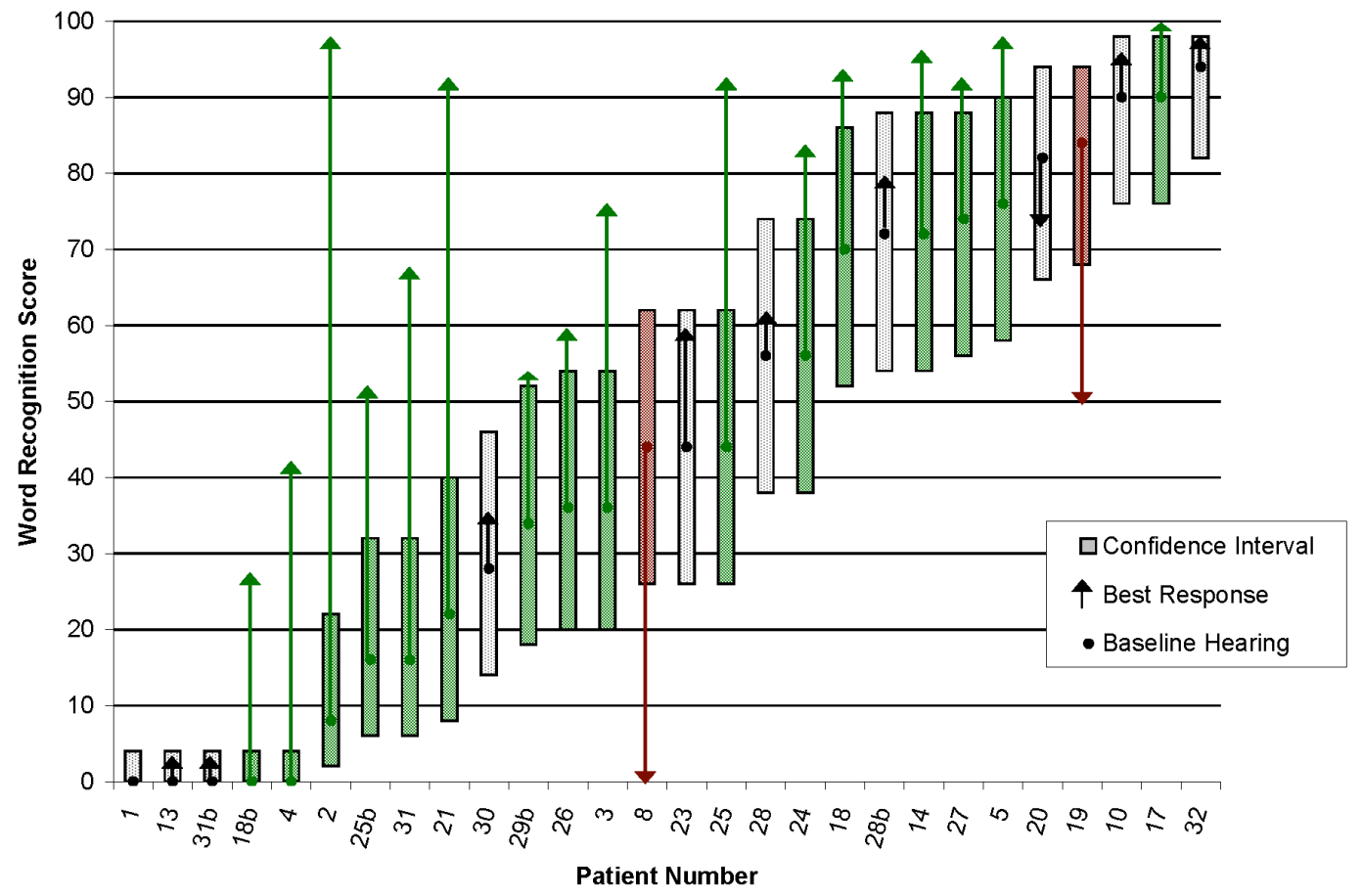

The best hearing responses are shown for the 23 evaluable target ears and the 5 evaluable contralateral ears. Each bar represents an individual ear. The absolute change in word recognition score from baseline is shown by the arrow. Arrows pointing upwards indicate hearing improvement. The 95\% critical difference (representing the statistical boundaries for hearing improvement and decline) are shown by colored boxes. Green arrows indicate hearing improvement; red arrows indicate hearing decline; grey arrows indicate stable hearing. 
Figure 3. Correlation between mean ADC value and change in tumor volume.

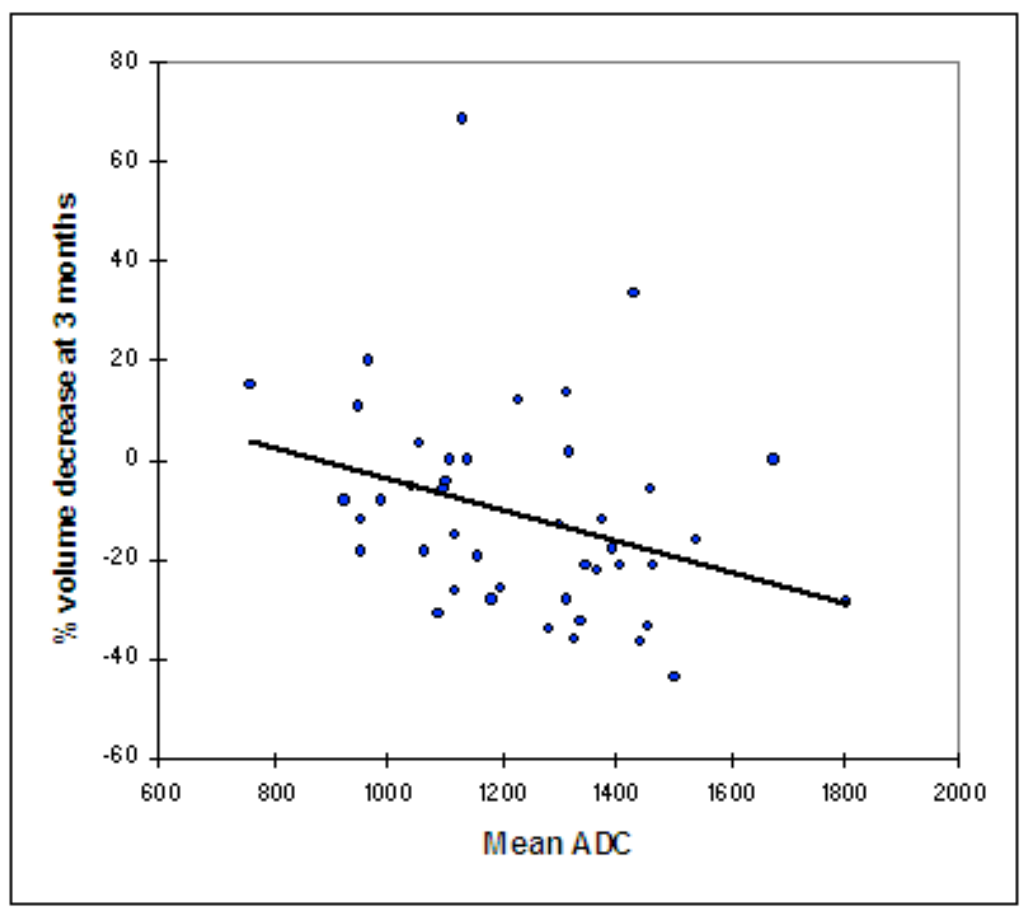

There was a significant correlation between mean ADC at baseline and subsequent decrease in tumor volume at 3 months for target vestibular schwannomas $(\mathrm{N}=29, \mathrm{r}=-0.458, \mathrm{p}=0.016)$ and all vestibular schwannomas $(\mathrm{N}=45, \mathrm{r}=-0.32, \mathrm{p}=0.036)$. 
Figure 4. Kaplan-Meier estimates of freedom from hearing loss and tumor growth.
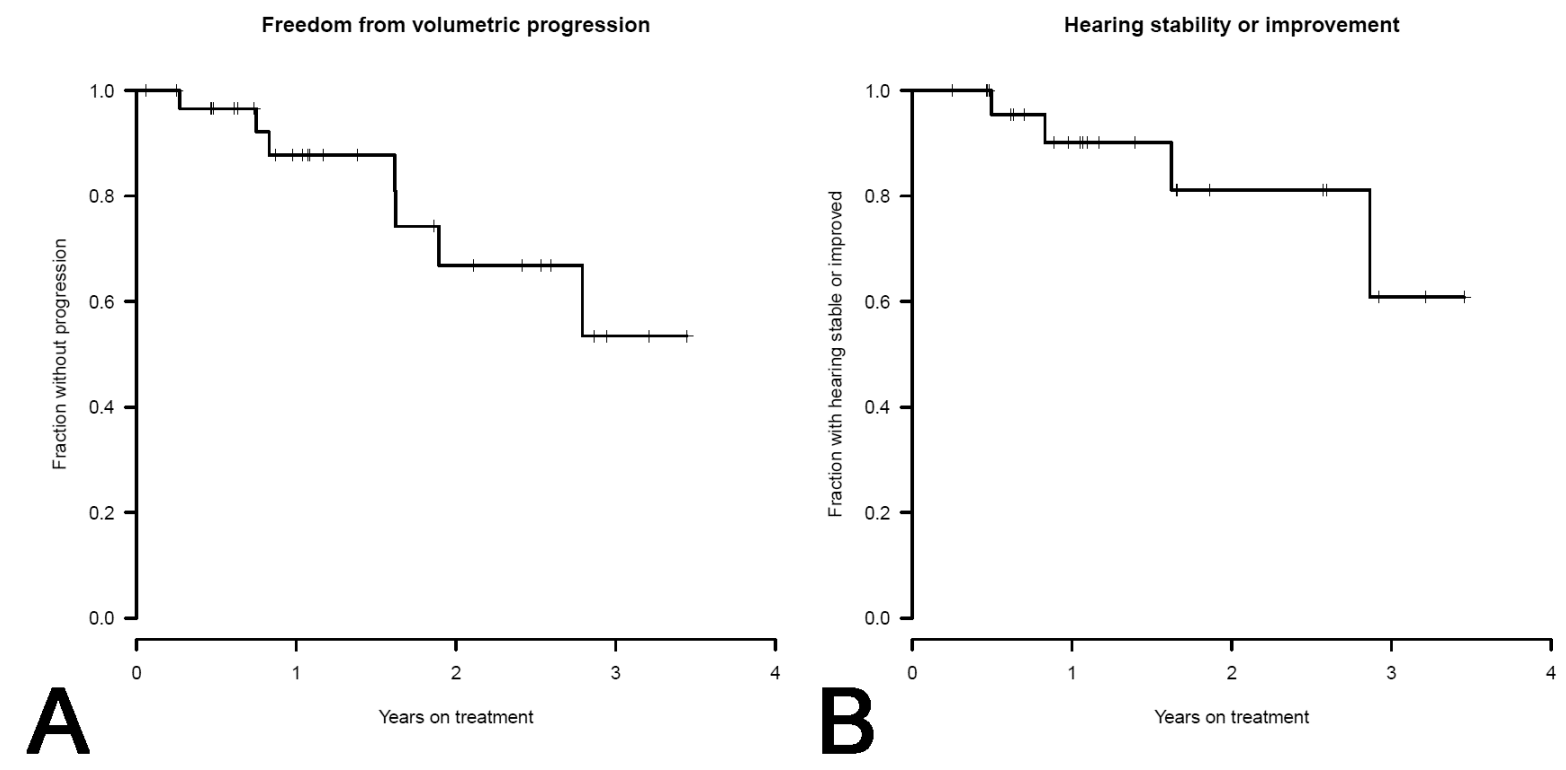

Panel A shows Kaplan-Meier estimates of freedom from hearing loss in patients; panel B shows Kaplan-Meier estimates of hearing from tumor growth in patients. The 1-year freedom from hearing loss was $90 \%$ and for tumor growth was $88 \%$. Hash marks indicate patient censoring. 
Supplemental Digital Content 1. Scattergram describing hearing function at baseline in 31 patients treated with bevacizumab based on the proposed 2011 AAO-HNS hearing classification.

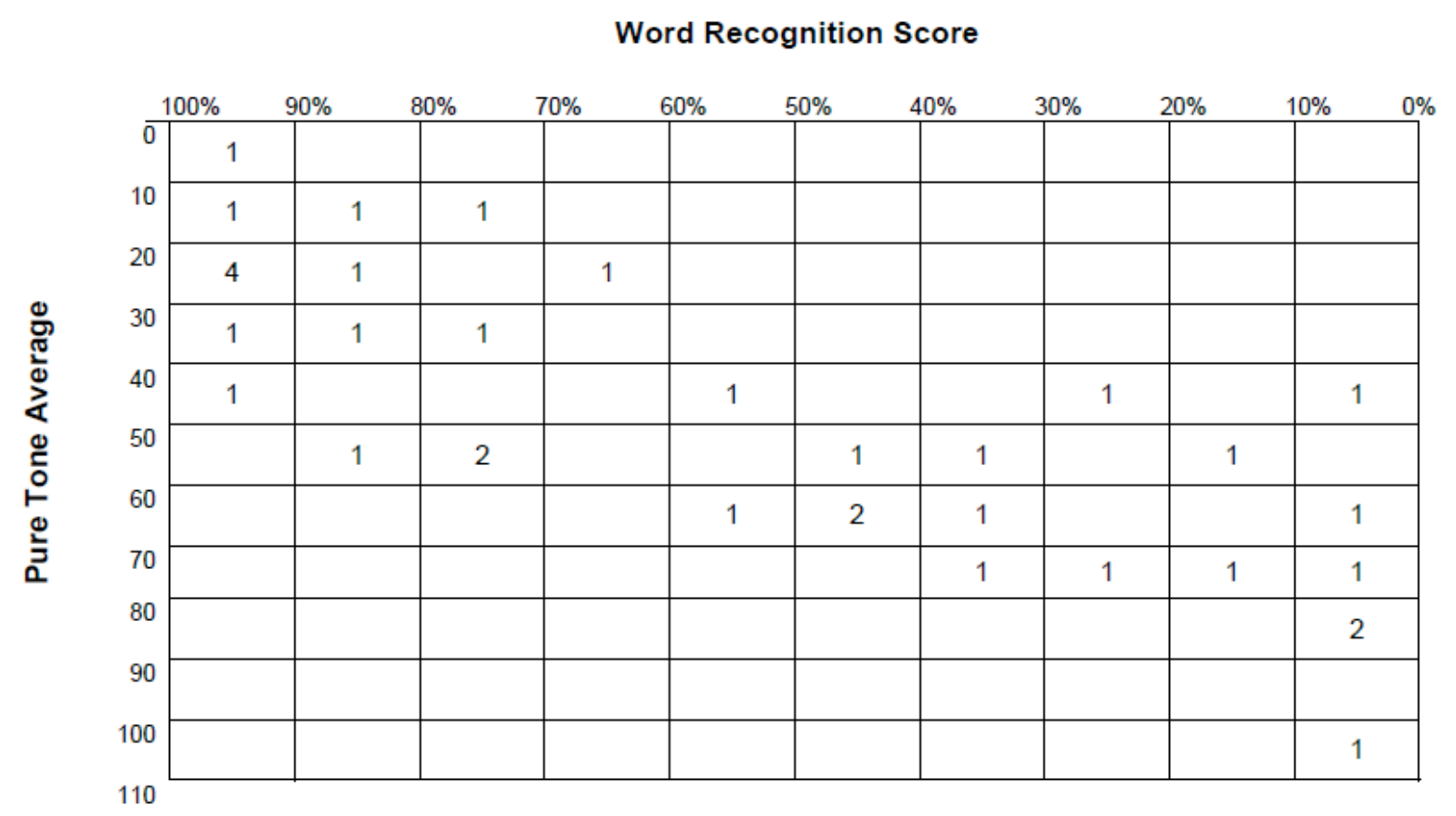

Word recognition score (in deciles) is shown on the top x-axis; pure tone average (in deciles) is shown on the y-axis. 
Supplemental digital content 2. AAO-HNS hearing classification for patients and baseline and at the time of last audiogram.

\begin{tabular}{|c|c|c|c|c|c|}
\hline \multirow{4}{*}{$\begin{array}{l}\text { Baseline } \\
\text { hearing } \\
\text { class }\end{array}$} & \multicolumn{5}{|c|}{ Post-treatment hearing class (response as of latest audiogram) } \\
\hline & & A & $\mathrm{B}$ & $\mathrm{C}$ & $\mathrm{D}$ \\
\hline & A & 5 & 4 & & \\
\hline & B & & 4 & 1 & \\
\hline & $\mathrm{C}$ & & & 3 & \\
\hline & $\mathrm{D}$ & 1 & 1 & 4 & 8 \\
\hline
\end{tabular}

6/31 (19\%) patients improved at least one class (gray boxes); 20/31 (65\%) did not change class (white boxes); $5 / 31$ (16\%) patients declined at least one class (red boxes). 